\title{
Controlled Synthesis of MnP Nanorods: The Effect of Shape Anisotropy on Magnetization
}

Kristy A. Gregg, ${ }^{\dagger}$ Susanthri C. Perera, ${ }^{\dagger}$ Gavin Lawes, ${ }^{\ddagger}$ Samuel Shinozaki, ${ }^{\dagger}$ Stephanie L. Brock $^{\dagger, *}$

Department of Chemistry $^{\dagger}$ and Department of Physics \& Astronomy ${ }^{\ddagger}$, Wayne State University, Detroit, Michigan 48202

\section{Supporting Information}

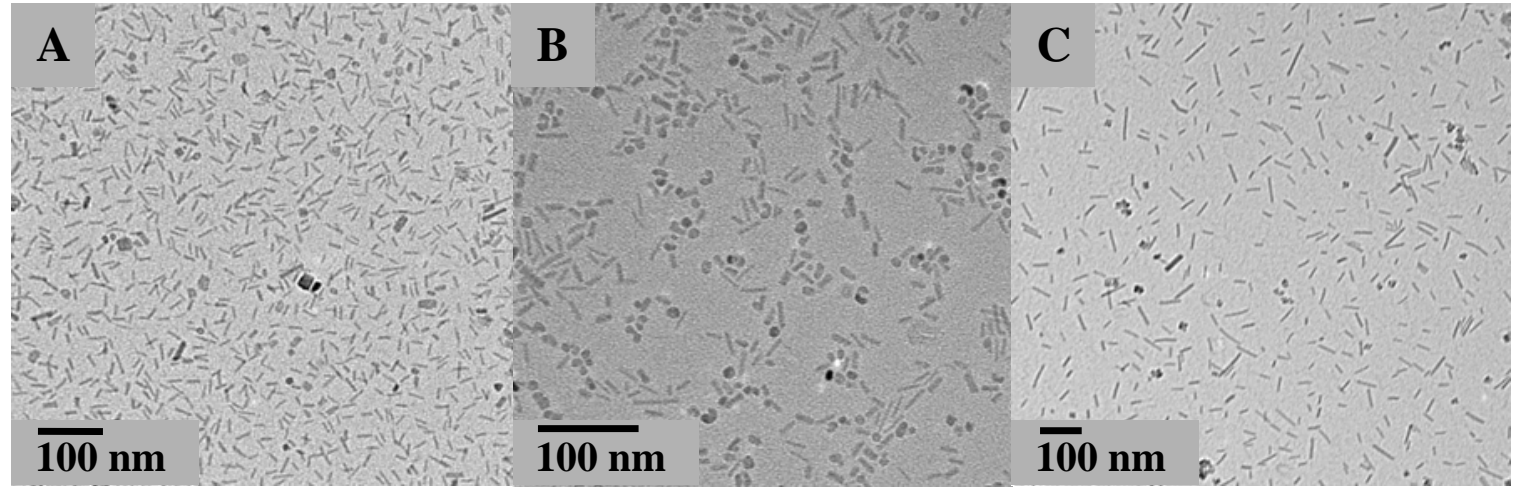

Figure S1. TEM images of MnP nanoparticles produced after changing the TOPO:TOP weight ratios to (A )10:90, (B) 25:75, and (C) 90:10. 

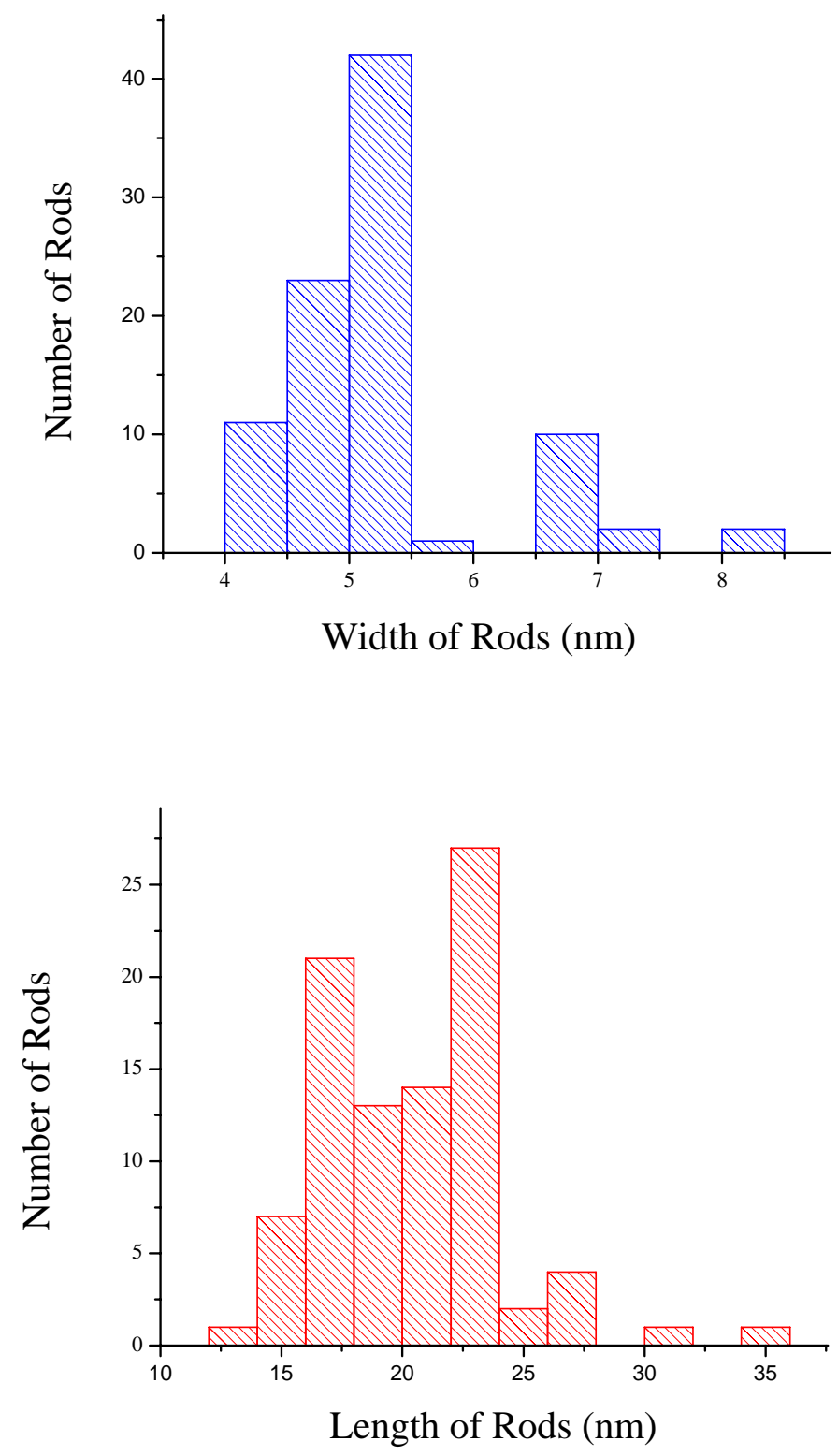

Figure S2. Histograms for the length and width of MnP nanorods (20.3 nm \pm 3.6 by 5.2 $n m \pm 0.89$ ). 


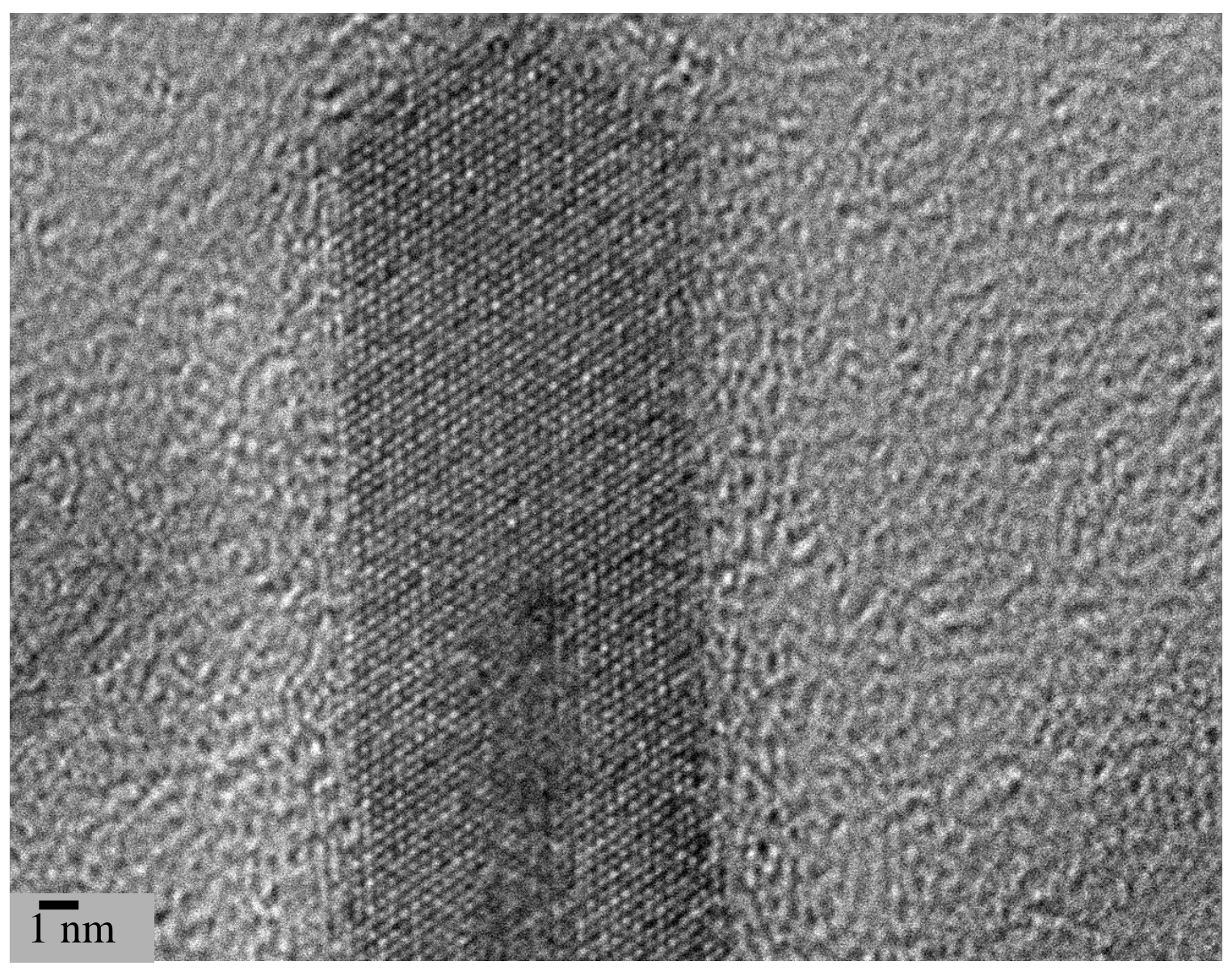

Figure S3. HRTEM of an individual MnP nanorod. The lattice planes running parallel to the rod growth direction are separated by a distance, $\mathrm{d}_{\mathrm{obs}}=2.797 \AA$, which corresponds to the (101) reflection of $\mathrm{MnP}\left(\mathrm{d}_{\text {calc }}=2.7966 \AA\right)$

Growth direction assignment: Parallel lattice fringes can be assigned to either the (200) or (101) reflections of MnP, with the latter suggesting growth along the b-axis. In the one case where off-axis fringes were observed (са. $20^{\circ}$ ), the $d$-spacing was found to be: $2.14 \AA$, which suggests indexing to either the $(021)$ or the $(211)$ reflections $(\mathrm{d}=$ $2.0252 \AA$ versus $2.0007 \AA$, respectively). The ideal angle from the b-axis can be obtained as shown below. Neither corresponds exactly to our observation, although the (211) is certainly closer. The best data for assignment of $\mathrm{b}$ as the growth direction clearly comes from the fringes parallel to the rod, and the qualitative observation that when fringes are not parallel to the rod axis, they contain a k-index.

(021): The plane cuts at $1 / 2$ along the b-axis (2.625 $\AA$ ) and 1 along the c-axis (3.17 $\AA$ ). The angle between $b$ and the hypotenuse of the corresponding right-triangle is the $\tan ^{-1}$ of $(3.17 / 2.625)=50^{\circ}$.

(211): The plane cuts at $1 / 2$ along a, 1 along b and c; and the computation can be achieved similar to the above case using a projection in the ab-plane or the bc-plane. Both give similar results, ca. $30^{\circ}$. 